Pacific Journal of Mathematic 


\title{
RADICAL PROPERTIES INVOLVING ONE-SIDED IDEALS
}

\author{
R. F. Rossa
}

A radical $P$ is called strongly right hereditary (srh) if $P(I)=I \cap P(R)$ for every right ideal $I$ of each (not necessarily associative) ring $R$ in a suitable universal class $W$. This is a one-sided version of the concept of a strongly hereditary radical class investigated by W.G. Leavitt and R.L. Tangeman. A discussion parallel to theirs is obtained including a construction of the minimal srh radical class in $W$ containing a given class. Srh radicals are related to a new radical construction obtained by modifying the lower radical construction of Tangeman and D. Kreiling.

1. Introduction. A class $M$ of not necessarily associative rings is called right hereditary if every right ideal of each ring in $M$ is also in $M$. Subring hereditary classes are defined in a corresponding way. A universal class is a homomorphically closed, subring hereditary class of rings. A radical $P$ of some universal class $W$ is strongly hereditary if for all $R \in W$ we have $P(I)=I \cap P(R)$ for all ideals $I$ of $R$, and strongly right hereditary (srh) if we have the same property for all right ideals $I$ of $R$. Strongly hereditary radicals have been studied by W. G. Leavitt [4] and R. L. Tangeman [6] using the following property (a) which may be satisfied by a class $M$ of rings in a universal class $W$ :

(a) If $J \in M$ is an ideal of an ideal $I$ of some $R \in W$, then the ideal $J^{\prime}$ of $R$ generated by $J$ is also in $M$. In $\S 2$, we obtain a parallel discussion of srh radicals using the following modification of (a):

( $\rho)$ If $J \in M$ is an ideal of a right ideal $I$ of $R \in W$, then the ideal $J^{\prime}$ of $R$ generated by $J$ is also in $M$.

In a universal class $W$, the lower radical determined by a class $M$ will be denoted by $L M$. In $\$ 3$, we introduce a new radical construction obtained by altering the construction of $L M$ given by Tangeman and Kreiling [3] at the limit ordinal step. A brief summary of their construction may be found in [5], whose notation we will continue to use. Our construction is related to property $(\rho)$ by Theorem 3.2.

For a class $M \leqq W$, the minimal right hereditary subclass of $W$ containing $M$ will be denoted by $G M$. Write $G_{1} M=M$ and, for $n \geqq 2, G_{n} M=\left\{R \in W: R\right.$ is a right ideal of some ring in $\left.G_{n-1} M\right\}$. Then $G M=\bigcup G_{n} M$, as in [5]. If $M=\{R\}$ consists of a single ring, we will omit braces and write, for example, $G_{n} M=G_{n} R$.

2. Srh radicals. The results of [4] and [6] all have one-sided 
versions. In particular, following [4, Theorem 1], we have.

Theonem 2.1. A right hereditary radical class $P \subseteq W$ is srh if and only if it has property $(\rho)$.

Next we show that property $(\rho)$ is inherited by the lower radical. Our proof is an adaptation of an unpublished proof by Tangeman of [6, Theorem 2.4].

THEOREM 2.2. Suppose $M \subseteq W$ is homomorphically closed and has property $(\rho)$. Then LM also satisfies $(\rho)$.

Proof. We will use the construction of $L M$ due to Tangeman and Kreiling and the notation of [5]. By hypothesis $M_{1}=M$ has property $(\rho)$. Let $\beta>1$ be an ordinal number and let $J$ be an ideal of a right ideal $I$ of a ring $R \in W$ such that $J \in M_{\beta}$. Let $J^{\prime}$ denote the ideal of $R$ generated by $J$. Suppose the classes $M_{\alpha}$ satisfy $(\rho)$ for all $\alpha<\beta$.

First suppose $\beta$ is a limit ordinal. Then $J=\bigcup J_{\gamma}$, where $\left\{J_{\gamma}\right\}$ is a chain of ideals of $J$ contained in $\bigcup_{\alpha<\beta} M_{\alpha}$. For each index $\gamma$, let $K_{r}$ be the ideal of $I$ generated by $J_{r}$. Then $J=\bigcup K_{r}$. By property $(\rho)$, each $K_{r} \in \bigcup_{\alpha<\beta} M_{\alpha}$. Now let $K_{\gamma}^{\prime}$ be the ideal of $R$ generated by $K_{r}$. By $(\rho)$ again, each $K_{\gamma}^{\prime} \in \bigcup_{\alpha<\beta} M_{\alpha}$. Since $J^{\prime} \supseteq K_{\gamma}$ for each $\gamma$ and $J^{\prime}$ is an ideal of $R, J^{\prime} \supseteq \bigcup K_{\gamma}^{\prime}$. On the other hand, since $\bigcup K_{\gamma}^{\prime}$ is an ideal of $R$ containing $\bigcup K_{r}=J$, we have $\bigcup K_{r}^{\prime} \supseteqq J^{\prime}$. Hence $J^{\prime}=\bigcup K_{r}^{\prime} \in M_{\beta}$.

If $\beta$ is not a limit ordinal, then $J$ has an ideal $K$ with $K$, $J / K \in M_{\beta-1}$. Now if $P \subseteq J$ is the ideal of $I$ generated by $K$, then $P \in M_{\beta-1}$ by property $(\rho)$. Moreover, $J / P \in M_{\beta-1}$ because $J / P$ is a homomorphic image of $J / K$ and $M_{\beta-1}$ is homomorphically closed [3, Lemma 2]. Now $P$ generates an ideal $Q$ of $R$ with $Q \in M_{\beta-1}$ by the inductive hypothesis. The ideal of $R / Q$ generated by $J+Q / Q$ is $J^{\prime} / Q$. Since $P \subseteq J \cap Q, J+Q / Q \simeq J / J \cap Q$ is a homomorphic image of $J / P$. Hence $J+Q / Q \in M_{\beta}$ and so, using $(\rho)$ again, $J^{\prime} / Q \in M_{\beta-1}$. Since $Q$, $J^{\prime} / Q \in M_{\beta-1}$, we have $J^{\prime} \in M_{\beta}$. The theorem follows by transfinite induction.

Let $E M=\left\{J^{\prime}: J\right.$ is an ideal of a right ideal of a ring $R \in W$, $J \in M$, and $J^{\prime}$ is the ideal of $R$ generated by $\left.J\right\}$. The homomorphic closure of $M$ will be denoted by $H M$. We have the following onesided version of [6, Corollary to Theorem 2.5].

THEOREM 2.3. If $W$ is a universal class and $M \cong W$, then there exists a unique minimal radical class in $W$ containing $M$ and satisfying property $(\rho)$.

Proof. Let $M_{1}^{*}=E H M$ and, inductively, $M_{n}^{*}=E H M_{n-1}^{*}$ for all integers $n>1$. Then $M^{*}=U M_{n}^{*}$ is a homomorphically closed class satis- 
fying $(\rho)$, so that $L M^{*}$ satisfies $(\rho)$ by Theorem 2.2 . On the other hand, any radical class which satisfies property $(\rho)$ and contains $M$ may be seen by induction to contain $M^{*}$ and hence $L M^{*}$.

EXAMPLE 2.1. The class $L M^{*}$ need not be hereditary when $M$ is hereditary. For let $K=G F(2)$ and let $R=\left\{\left(\begin{array}{ll}a & 0 \\ b & 0\end{array}\right): a, b \in K\right\}$. We identify isomorphic rings; thus $K \simeq\left\{\left(\begin{array}{ll}a & 0 \\ 0 & 0\end{array}\right): a \in K\right\}$ is a right ideal of $R$ with $K^{\prime}=R . \quad R$ has the ideal $I=\left\{\left(\begin{array}{ll}0 & 0 \\ b & 0\end{array}\right): b \in K\right\}$. Let $W=$ $\{R, K, I, 0\}, M=\{K, 0\}$. Then $M$ is hereditary, while $L M^{*}=M^{*}=$ $\{R, K, 0\}$ is not.

As in [6, Corollary (c)] and [8, Corollary 2.7], we also have

THeOREM 2.4. If $W$ is a universal class and $M \subseteq W$, then there is a unique minimal srh radical class in $W$ containing $M$.

Proof. Define $\bar{M}_{1}=E G H M$ and, for all $n>1, \bar{M}_{n}=E G H \bar{M}_{n-1}$. Then $\bar{M}=\mathrm{U} \bar{M}_{n}$ is the minimal subclass of $W$ which contains $M$, satisfies property $(\rho)$ and is right hereditary and homomorphically closed. Then $L \bar{M}$ is the desired srh radical class, for by Theorem 2.2 $L \bar{M}$ has property $(\rho)$ and by [5, Theorem 2], $L \bar{M}$ is right hereditary. Thus by Theorem 2.1, $L \bar{M}$ is srh; it is again easy to see that $L \bar{M}$ is minimal.

We turn to a consideration of two properties similar to property $(\rho)$.

THEOREM 2.5. Let $M$ be a class of rings satisfying property $(\rho)$. For all $R \in W$, if $I \in M$ is in $G R$, then the ideal $I^{\prime}$ of $R$ generated by $I$ is also in $M$.

Proof. The theorem is trivially satisfied when $I \in M \cap G_{1} R$. Thus for induction assume for all $R \in W$ and all $I \in M \cap G_{n} R$ that $I^{\prime} \in M$, where $I^{\prime}$ is the ideal of $R$ generated by $I$. Let $K \in M \cap G_{n+1} R$ so that $K \in M \cap G_{n} J$ for some right ideal $J$ of $R$. By induction $K^{*} \in M$ where $K^{*}$ is the ideal of $J$ generated by $K$. But then $(\rho)$ implies $K^{\prime}=K^{* \prime} \in M$.

CoROLlaRY. Property $(\rho)$ is equivalent to the following property $\left(\rho^{\prime}\right)$ :

$\left(\rho^{\prime}\right)$ If $J \in M$ is a right ideal of a right ideal $I$ of $R \in W$, then the ideal $J^{\prime}$ of $R$ generated by $J$ is also in $M$.

Consider the following property $(\sigma)$ : If $J \in M$ is a right ideal of $R$, then the ideal of $J^{\prime}$ of $R$ generated by $J$ is also in $M$. In general this property is not inherited by $L M$ as may be seen from the following example (for which we thank the referee). 
EXAMPLE 2.2. Let $K$ be generated over $G F(2)$ by $x, y, z$ where $x^{2}=y^{2}=0, x y=y x=x, y z=x z=z y=y$, and $z x=z^{2}=z$. Then $I=$ $\{0, x\}$ is an ideal of $R=\{0, x, y, x+y\}$ and $R$ is the only proper right ideal of $K$. Also $K$ is simple so that $R^{\prime}=K$. For the universal class $W$ consisting of $K$ and all its subrings, the class $M=\{0, I\}$ has property $(\sigma)$. However, $L M$ does not have the property since $R \in L M$ whereas $R^{\prime}=K \notin L M$.

For semisimple classes, we have the following one-sided version of [1, Theorem 4.1] and [6, Theorem 3.1], which we state without proof.

THEOREM 2.6. $Q$ is a semisimple class for a radical class $P$ with property $(\rho)$ if and only if $Q$ has properties (b), (c), and (d) of [1, Theorem 4.1] and is right hereditary.

In general it cannot be expected that semisimple subideals will generate semisimple ideals, as in property (a). Indeed, if the radical class is not hereditary, a semisimple subideal may even generate a radical ideal. We give two examples using well-known radicals in the universal class of associative rings.

ExAmple 2.3. Let $A$ be a ring isomorphic to the ring of even integers with generator $a$. Let $B=\{0, x\}, C=\{0, y\}$ be zero rings of order two. Let $I=A \oplus B$, and form $R$ by adjoining $C$ to $I$ in such a way that the additive group of $R$ is $I+C$ (direct sum), (na)y= $y(n a)=n x$ for all integers $n$, and $x y=y x=0$. Then $I$ is an ideal of $R$ and $A$ is a nil-semisimple ideal of $I$, but $A^{\prime}=I$ has the nil ideal $B$.

EXAmple 2.4. Let $A$ be the zero ring whose additive group is $Z_{p}(\infty)$ and let $B$ be the ring of polynomials of degree $\geqq 1$ over $G F$ (2). Define the commutative ring $R$ as follows. The additive group of $R$ is the direct sum $A+B$; the multiplication within $A$ and $B$ is as usual, and we define $\left(\alpha / p^{n}\right) x^{i}=\alpha / p^{n+i}$, extending this multiplication to $R$ in the natural way.

Let $I$ be the subring of $A$ of order $p$. Thus $I$ is an ideal of $A$, and the ideal $I$ generates in $R$ is $A$. In the upper radical of the class of all simple rings (see [2, page 14]), $I$ is semisimple and $A$ is radical.

3. Radical constructions involving one-sided ideals. Let $M$ be any class contained in a universal class $W$. We will construct a class $\Delta M$ (depending of course on the universal class $W$ ) by modifying the radical construction of [3]. Briefly, let $\Delta_{1} M$ be the homomorphic closure of $M$. We proceed inductively to define a class $\Delta_{\beta} M$ for each ordinal number $\beta$. If $\beta-1$ exists, let $\Delta_{\beta} M=\{R \in W: R$ has an ideal $J$ such that $\left.J, R / J \in \Delta_{\beta-1} M\right\}$. If $\beta$ is a limit ordinal, define $R \in$ 
$\Delta_{\beta} M$ if and only if $R$ is the union of a chain $\left\{I_{\gamma}\right\}$ of right ideals of $R$ such that each $I_{\gamma} \in \mathrm{U}_{\alpha<\beta} \Delta_{\alpha} M$. Finally, let $\Delta M=\mathrm{U}_{\beta} \Delta_{\beta} M$.

By modifying suitably the proof of [3, Theorem 2] we have

TheOREM 3.1. $\Delta M$ is a radical class.

The corresponding construction using left ideals yields a radical class we will call $\Lambda M$.

THEOREM 3.2. If $M$ is homomorphically closed and has property $(\rho)$, then $L M=\Delta M$.

Proof. Since $M \subseteq \Delta M$ and $\Delta M$ is radical, $L M \subseteq \Delta M$. Thus assume for induction that, for $\beta$ a given ordinal, $\Delta_{\infty} M \subseteq L M$ for all $\propto<\beta$. If $R \in M_{\beta}$ is a nonlimit ordinal then $I, R / I \in \Delta_{\beta-1} M \leqq L M$, so that $R \in L M$. If $\beta$ is a limit ordinal then $R=I_{r}$ for some chain $\left\{I_{r}\right\}$ of right ideals contained in $\bigcup_{\alpha<\beta} \Delta_{\propto} M \subseteq L M$. But by Theorem 2.2, $L M$ has property $(\rho)$. Thus if $I_{r}^{\prime}$ is the ideal of $R$ generated by $I_{r}$, then $I_{r}^{\prime} \in L M$ and so $R=\bigcup I_{r}^{\prime} \in L M$. Thus $\Delta_{\beta} M \leqq L M$ and so $\Delta M \cong L M$.

This is not a necessary condition, for let $M$ be the nil radical class in the universal class of associative rings. Then $M=L M=\Delta M$, but $M$ does not have property $(\rho)$ by Theorem 2.6 because the nilsemisimple rings do not form a right hereditary class.

Even in the associative case, $\Delta M$ and $A M$ may be unequal.

EXAMPLE 3.1. Let $R$ be the associative algebra over the field $G F(2)$ generated by a countable number of symbols $\left\{x_{i}: i=1,2, \cdots\right\}$ subject to the relations $x_{i} x_{j}=x_{j}$ for all $i, j$. For each $n$, let $I_{n}$ be the left ideal generated by $\left\{x_{1}, \cdots, x_{n}\right\}$. Then $M=\left\{I_{n}: n=1,2, \cdots\right\}$ is a chain of left ideals of $R$ and $R=I_{n}$, so that $R \in A M$. Since $R$ has no proper right ideals and $R \notin M_{1}=\Delta_{1} M, R$ cannot be in $\Delta M$.

\section{REFERENCES}

1. E. P. Armendariz, Closure properties in radical theory, Pacific J. Math., 26 (1968), 1-7.

2. N. J. Divinsky, Rings and Radicals, Univ. of Toronto Press, 1965.

3. D. Kreiling and R. L. Tangeman, Lower radicals in nonassociative rings, J. Austral. Math. Soc., (to appear).

4. W. G. Leavitt, Strongly hereditary radicals, Proc. Amer. Math. Soc., 21 (1969), 703-705.

5. R. F. Rossa, More properties inherited by the lower radical, Proc. Amer. Math. Soc., 33 (1972), 247-249.

6. R. L. Tangeman, Strong heredity in radical classes, Pacific J. Math., 42 (1972), 259-266.

Received September 28, 1972 and in revised form December 12, 1972. 



\section{PACIFIC JOURNAL OF MATHEMATICS}

\section{EDITORS}

RICHARD ARENS (Managing Editor)

University of California

Los Angeles, California 90024

\section{R. A. Beaumont}

University of Washington

Seattle, Washington 98105

\section{J. DuGundJI*}

Department of Mathematics

University of Southern California

Los Angeles, California 90007

D. Gilbarg and J. Milgram

Stanford University

Stanford, California 94305

ASSOCIATE EDITORS
E. F. BECKENBACH
B. H. NeumanN
F. WOLF
K. YoSHIDA

\section{SUPPORTING INSTITUTIONS}

UNIVERSITY OF BRITISH COLUMBIA

CALIFORNIA INSTITUTE OF TECHNOLOGY

UNIVERSITY OF CALIFORNIA

MONTANA STATE UNIVERSITY

UNIVERSITY OF NEVADA

NEW MEXICO STATE UNIVERSITY

OREGON STATE UNIVERSITY

UNIVERSITY OF OREGON

OSAKA UNIVERSITY

\author{
UNIVERSITY OF SOUTHERN CALIFORNIA \\ STANFORD UNIVERSITY \\ UNIVERSITY OF TOKYO \\ UNIVERSITY OF UTAH \\ WASHINGTON STATE UNIVERSITY \\ UNIVERSITY OF WASHINGTON \\ AMERICAN MATHEMATICAL SOCIETY \\ NAVAL WEAPONS CENTER
}

The Supporting Institutions listed above contribute to the cost of publication of this Journal, but they are not owners or publishers and have no responsibility for its content or policies.

Mathematical papers intended for publication in the Pacific Journal of Mathematics should be in typed form or offset-reproduced, (not dittoed), double spaced with large margins. Underline Greek letters in red, German in green, and script in blue. The first paragraph or two must be capable of being used separately as a synopsis of the entire paper. Items of the bibliography should not be cited there unless absolutely necessary, in which case they must be identified by author and Journal, rather than by item number. Manuscripts, in duplicate if possible, may be sent to any one of the four editors. Please classify according to the scheme of Math. Rev. Index to Vol. 39. All other communications to the editors should be addressed to the managing editor, or Elaine Barth, University of California, Los Angeles, California, 90024.

50 reprints are provided free for each article; additional copies may be obtained at cost in multiples of 50 .

The Pacific Journal of Mathematics is issued monthly as of January 1966. Regular subscription rate: $\$ 48.00$ a year (6 Vols., 12 issues). Special rate: $\$ 24.00$ a year to individual members of supporting institutions.

Subscriptions, orders for back numbers, and changes of address should be sent to Pacific Journal of Mathematics, 103 Highland Boulevard, Berkeley, California, 94708.

\section{PUBLISHED BY PACIFIC JOURNAL OF MATHEMATICS, A NON-PROFIT CORPORATION}

Printed at Kokusai Bunken Insatsusha (International Academic Printing Co., Ltd.), 270, 3-chome Totsuka-cho, Shinjuku-ku, Tokyo 160, Japan.

* C. R. DePrima California Institute of Technology, Pasadena, CA 91109, will replace J. Dugundji until August 1974.

Copyright (C) 1973 by

Pacific Journal of Mathematics

All Rights Reserved 


\section{Pacific Journal of Mathematics}

\section{Vol. 49, No. $2 \quad$ June, 1973}

Wm. R. Allaway, On finding the distribution function for an orthogonal polynomial

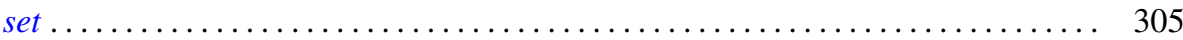

Eric Amar, Sur un théorème de Mooney relatif aux fonctions analytiques bornées... . 311

Robert Morgan Brooks, Analytic structure in the spectrum of a natural system . . . . 315

Bahattin Cengiz, On extremely regular function spaces . . . . . . . . . . . . . . 335

Kwang-nan Chow and Moses Glasner, Atoms on the Royden boundary . . . . . . . . . 339

Paul Frazier Duvall, Jr. and Jim Maxwell, Tame $Z^{2}$-actions on $E^{n} \ldots \ldots \ldots \ldots \ldots . .349$

Allen Roy Freedman, On the additivity theorem for $n$-dimensional asymptotic

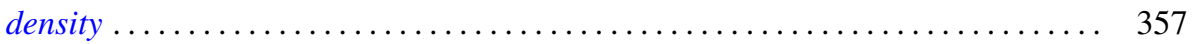

John Griffin and Kelly Denis McKennon, Multipliers and the group $L_{p}$-algebras . . . 365

Charles Lemuel Hagopian, Characterizations of $\lambda$ connected plane continua ....... 371

Jon Craig Helton, Bounds for products of interval functions . . . . . . . . . . . 377

Ikuko Kayashima, On relations between Nörlund and Riesz means . . . . . . . . . . 391

Everett Lee Lady, Slender rings and modules . . . . . . . . . . . . . . . . . . 397

Shozo Matsuura, On the Lu Qi-Keng conjecture and the Bergman representative

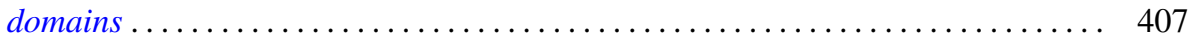

Stephen H. McCleary, The lattice-ordered group of automorphisms of an $\alpha$-set . . . 417

Stephen H. McCleary, o-2-transitive ordered permutation groups .......... 425

Stephen H. McCleary, o-primitive ordered permutation groups. II . . . . . . . . . 431

Richard Rochberg, Almost isometries of Banach spaces and moduli of planar

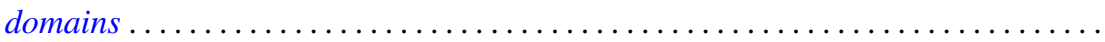

R. F. Rossa, Radical properties involving one-sided ideals . . . . . . . . . . . . . 467

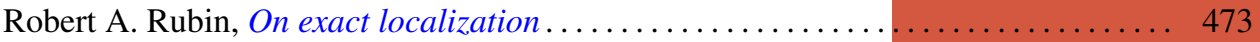

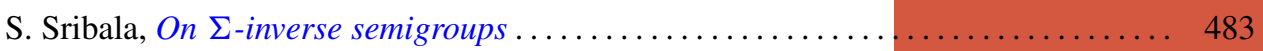

H. M. (Hari Mohan) Srivastava, On the Konhauser sets of biorthogonal polynomials suggested by the Laguerre polynomials ...................... 489

Stuart A. Steinberg, Rings of quotients of rings without nilpotent elements . ...... 493

Daniel Mullane Sunday, The self-equivalences of an $H$-space . . ............ 507

W. J. Thron and Richard Hawks Warren, On the lattice of proximities of $\check{C} e c h$

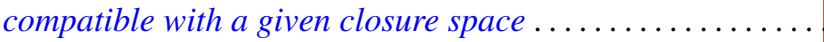

Frank Uhlig, The number of vectors jointly annihilated by two real quadratic forms determines the inertia of matrices in the associated pencil .

Frank Uhlig, On the maximal number of linearly independent real vectors annihilated simultaneously by two real quadratic forms ..............

Frank Uhlig, Definite and semidefinite matrices in a real symmetric matrix pencil . . 561

Arnold Lewis Villone, Self-adjoint extensions of symmetric differential operators . . . 569

Cary Webb, Tensor and direct products . ....................... 579

James Victor Whittaker, On normal subgroups of differentiable

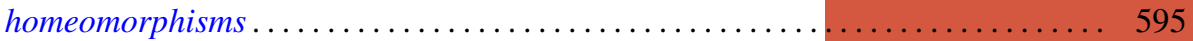

Jerome L. Paul, Addendum to: "Sequences of homeomorphisms which converge to

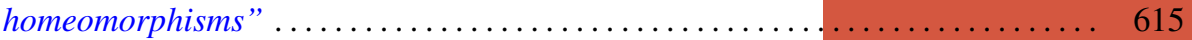

David E. Fields, Correction to: “Dimension theory in power series rings” ........ 616

Peter Michael Curran, Correction to: “Cohomology of finitely presented groups”. . . 617

Billy E. Rhoades, Correction to: “Commutants of some Hausdorff matrices” ...... 617

Charles W. Trigg, Corrections to: "Versum sequences in the binary system” ...... 619 